\title{
Perception of emotional facial expressions in individuals with high Autism-spectrum Quotient (AQ)
}

\author{
Ervin Poljac*
}

\begin{abstract}
Autism is characterized by difficulties in social interaction, communication, restrictive and repetitive behaviours and specific impairments in emotional processing. The present study employed The Autism Spectrum Quotient (Baron-Cohen et al. 2006) to quantify autistic traits in a group of 260 healthy individuals and to investigate whether this measure is related to the perception of facial emotional expressions. The emotional processing of twelve participants that scored significantly higher than the average on the AQ was compared to twelve participants with significantly lower AQ scores. Perception of emotional expressions was estimated by The Facial Recognition Task (Montagne et al. 2007). There were significant differences between the two groups with regard to accuracy and sensitivity of the perception of emotional facial expressions. Specifically, the group with high AQ score was less accurate and needed higher emotional content to recognize emotions of anger, disgust, happiness and sadness. This result implies a selective impairment that might be helpful in understanding the psychopathology of autism spectrum disorders.
\end{abstract}

Keywords: Autism spectrum quotient, Face perception, Emotion recognition, Facial expression, Affective processing

\footnotetext{
Corresponding author: Ervin Poljac; Laboratory of Experimental Psychology, University of Leuven, Leuven, Belgium; e-mail: Ervin.Poljac@psy.kuleuven.be
} 


\section{Introduction}

Interpretation of emotional states of others is essential for establishing normal interpersonal relationships. Facial expressions convey very strong non-verbal cues of emotional states and their perception and interpretation is crucial in social interactions. Many psychiatric and neurological disorders are accompanied by impaired emotional processing (Lembke and Ketter, 2002; McClure, Pope, Hoberman, Pine, \& Leibenluft, 2003; Green, Turner, \& Thompson, 2004; Venn et al., 2004; Montagne, Kessels, Wester, \& de Haan, 2006a). Depression or social anxiety, for instance, are known to disrupt the perception and memory of emotional cues, causing a lower sensitivity for the negative facial expressions of anger and disgust (Montagne, Schutters, Westenberg, Kessels, \& de Haan, 2006b).

Autism is a disorder characterized by specific impairments in social interaction, communication and restrictive and repetitive, stereotyped behavior (American Psychiatric Association, 2000). These deficits have been repeatedly demonstrated in previous research and in clinical practice (e.g. Baron-Cohen, Tager-Flusberg \& Cohen, 1993; Dawson, Meltzhoff, Osterling, Rinaldi \& Brown, 1998). With regard to their possible origins, there is evidence that autism is related to early dysfunction of brain areas responsible for social cognition and emotional processing (Baron-Cohen, et al., 1999; Dawson, 1996). In particular, amygdala has been related to an altered emotional function in autism, having not only atypical function, but also some structural differences compared to non-patients. In support to this argument, Kleinhans et al. (1999) showed a reduced effect of adaptation in the amygdala of autistic individuals due to repeated exposure to faces. The observed lack of adaptation to faces (or the hyperactivity of amygdala, as the authors refer to it) might be an important clue for the deviating processing of faces in autism and used as evidence that autistic people do not treat faces differently than other objects (Rosset et al., 2008). In specific, the ability to use or understand facial information is claimed to be an important deficit in autism (Baron-Cohen, 1993; Frith, 1989) with emotional functional deficit as a consequence. Hence, these impairments are often 
assumed responsible for distinctive disorders in emotional processing, such as interpreting emotional expressions.

A number of studies provide evidence that the processing of basic emotions is indeed altered in autism (Bolte \& Poustka, 2003; Celani, Battacchi, \& Arcidiacono, 1999; Davies, Bishop, Manstead \& Tantam, 1994), however, other studies have failed to find such decline (BaronCohen, Jolliffe, Martimore, \& Robertson, 1997; Grossman, Klein, Carter, \& Volkmar, 2000; Ogai et al., 2003). One possible explanation for this inconsistency might be related to the employed stimuli: Frequently used (static) pictures of fully expressed emotions might have resulted in ceiling effects and thus were not sensitive enough to detect difficulties in emotional processing. Cartoon-like presentations of facial expressions usually contain exaggerated representations of emotions, and autistic children seem to process this information in a very typical way, similar to controls (Rosset et al., 2008). However, when it comes to real faces, there are noticeable differences. Hence, more subtle emotional facial expressions might reveal possible differences in the processing of emotions. Recently, Montagne, Kessels, De Haan, \& Perrett, (2007) developed a novel test for estimating the accuracy and sensitivity of emotional processing, the Emotion Recognition Task (ERT). This test makes it possible to estimate emotional processing at different levels of facial emotional expressions and it is more sensitive for possible subtle differences in processing. Moreover, because the test involves dynamic presentations, it is likely more ecologically valid compared to static presentations, as it involves more realistic presentations of emotions.

The current study examined the recognition of emotional facial expressions in individuals with a high Autism-spectrum Quotient (AQ, Baron-Cohen et al. 2006) score. These are healthy individuals who share common characteristics with people diagnosed with autism. The perception of what are generally agreed to be the six basic or universal emotions: anger, disgust, fear, happiness, sadness and surprise (Ekman, 1999), was examined, both in terms of accuracy and the sensitivity. Because of frequently observed impairments in emotional processing in individuals with autism, a decline in the accuracy and sensitivity of the 
recognition of these emotions was expected to be observed in the individuals scoring high on the AQ.

\section{Methods}

\section{Participants}

Two hundred sixty students from the undergraduate programs at the International University of Sarajevo took part in the current study. The study has been carried out in accordance with the ethical standards laid down in the 1964 Declaration of Helsinki. Participation was voluntary and all the subjects gave their informed consent prior to the inclusion into the study.

\section{Autism-spectrum Quotient}

To quantify the amount of autistic traits in a group of 260 students, the Autism-spectrum Quotient (Baron-Cohen et al. 2006) was employed. The AQ was published in 2001 by autism expert Simon Baron-Cohen and his colleagues at the Autism Research Centre at the University of Cambridge. The test consists of fifty statements, each with four (forced) choices to indicate whether subjects 'definitely agree', 'slightly agree', 'slightly disagree', or 'definitely disagree' with each statement. Eighty percent of people with Asperger syndrome has a score between 32 and 50, while in a control group only two percent of people got that score. The scales of the test include willingness of adequate social interaction and interest, the degree of repetition, attention to detail, degree of empathy and imagination. The distribution of the participants' AQ scores in the present study resembled normal, with the average score of 14.95 (SD 5.45).

\section{Emotion Recognition Task}

Twelve participants with a score well above the average AQ score $(29 \pm 3.5)$ and equal number of students that scored significantly below the average $(6 \pm 2.3)$ were selected. There were no significant differences in age between the two groups. The participants performed The Emotion Recognition Task, a computer task used to evaluate the emotional 
processing i.e. the recognition of emotional facial expressions, developed by Montagne et al. (2007). Colour pictures of the faces of four actors (two males, two females), who posed six emotional expressions (anger, disgust, fear, happiness, sadness and surprise) and a neutral face, were utilized to create computer-generated morphs of 19 intermediate images between a neutral face ( $0 \%$ emotion) and a full-blown expression (100\% emotion). The task was developed from algorithms designed by Benson and Perrett (1991). In the morphed images, both the dimension of shape and texture underwent gradual transitions. These images were used to construct short video clips that incrementally increase in the degree of intensity of the expression by $10 \%$ steps. For each actor, and each of the six emotions, there were nine video clips (0-20, 0-30, 0-40, 0-50, 0-60, 070, 0-80, 0-90, 0-100\%; Fig. 1).



Figure 1. The Emotion Recognition Task shows (A) the gradual transition from neutral $(0 \%)$ to the full-blown emotion of happiness (100\%) in steps of $20 \%$, and (B) the gradual transition from neutral $(0 \%)$ to the full-blown emotion of anger $(100 \%)$ in steps of $20 \%$.

\section{Benton Facial Recognition Test}

The short form of the Benton Facial Recognition Test (Benton et al., 1994) was administered to assess perception of neutral faces. This test is able to detect possible generalized disorders of face perception and it consists of a booklet with a single target face on each page, and below it, a set of six faces. In the first six trials, an identical face has to be selected among five decoys. In the remaining 7 trials, three different views 
(changed in orientation or lighting conditions compared to the target photograph) have to be distinguished from three incorrect alternatives. The faces are unfamiliar and physically similar, without spectacles or facial hair. The maximum number of correctly identified faces is 27 .

\section{Beck Depression Inventory (BDI) scores}

The BDI (Beck et al., 1961), a 21-item self-report questionnaire, was used to measure the presence and the intensity of depressive symptoms in the participants.

\section{Procedure}

Each participant completed the AQ prior to The Emotion Recognition task. The task started with four practice trials, followed by the main experiment. The clips entailing morphed images of emotional expressions were presented in an incremental order, starting with $20 \%$ intensity levels and ending with a $100 \%$ expression. Thus, subjects first saw the 24 presentations of the lowest level of emotional expression (0$20 \%$ ), followed by the second set of 24 trials with video clips from $0 \%$ to $30 \%$, and so on until they reached the final sequence of clips in which the neutral face changes into a full-blown expression. Within each level, the order of presentation of emotions and actors was randomized. On each trial, subjects were required to make a forced choice between one of six emotional expression labels that were displayed on the screen during the time of presentation. The duration of the animation in the video clips varied between approximately $0.5 \mathrm{sec}$ (low intensity) and $2 \mathrm{sec}$ (high intensity). The moving expressions were created in an artificial manner but appear very similar to natural expressions. After the animation, the last frame remained on the screen until the subject made a response, at which time the new trial was automatically initiated. In total each participant performed 216 trials. There was no time restriction. The procedure was identical for both groups.

\section{Results}

The performance of 12 students with high AQ scores on The Emotion Recognition Task was compared to the performance of 12 
students of low-score group. The study used a mixed-factor design, with the group as a between subjects factor with two levels (high AQ group vs. low AQ group), and two within-subjects factors: The type of emotion (with six levels: anger, disgust, fear, happiness, sadness, surprise) and emotion intensity (with nine levels 0-20, 0-30, 0-40, 0-50, 0-60, 0-70, 080, 0-90, 0-100\%).

\section{Control tasks}

There were no significant differences between the groups with regard to BDI scores $(\mathrm{p}=0.86)$. In addition, the short form of the Benton Facial Recognition Test did not reveal significant differences between the high and low AQ groups with regard to the perception of neutral faces. The accuracy and sensitivity of emotion perception

Two different measures were used to estimate subjects' performance; accuracy and sensitivity. Recognition accuracy was defined as the percentage of correct answers for each emotional expression at each intensity level. Perceptual sensitivity for a specific emotion was defined as the level of emotional intensity that was required for the correct identification of this emotion. The perceptual sensitivity measure was calculated as the percentage of required intensity from which level onwards the emotion was consistently correctly identified.

Increased intensity of emotional expression resulted in a higher recognition rate of all emotions in both groups, although the slopes differ between individual emotions (Figure 2). The percentage of correct answers for each emotional expression at each intensity level was analyzed with a $9 \times 2$ repeated measures analysis of variance (ANOVA), general linear model (GLM) with group (high AQ group vs. low AQ) as between-subject variable and intensity level as within-subject variable. Overall, the accuracy fluctuated for different emotions $(F(5,19)=18.39$, $\mathrm{p}<.001)$. Most accurate recognition performance was observed for the emotion happiness (significantly more accurate than all other emotions; all F-values $>19.09, \mathrm{p}<.001)$, followed by anger, disgust and surprise. Montagne et al. (2005) also found the lowest thresholds for happiness, and the highest for fear and sadness. There were significant differences between the high and low AQ groups with respect to the two measures (Figure 3). The high AQ group was less accurate $(F(5,19)=27.05 ; \mathrm{p}<$ 
$.001)$ and on the average required more expression in the face to correctly identify the emotions of anger $(\mathrm{F}(1,22)=8.30 ; \mathrm{p}<.001)$, disgust $(\mathrm{F}(1$, 22) $=15.06$;

$\mathrm{p}<.001)$, happiness $(\mathrm{F}(1,22)=5.68 ; \mathrm{p}=.02)$, and sadness $(\mathrm{F}(1,22)=$ $9.63 ; \mathrm{p}<.001)$. The observed pattern is consistent with the results of the accuracy measure and in line with previous findings.
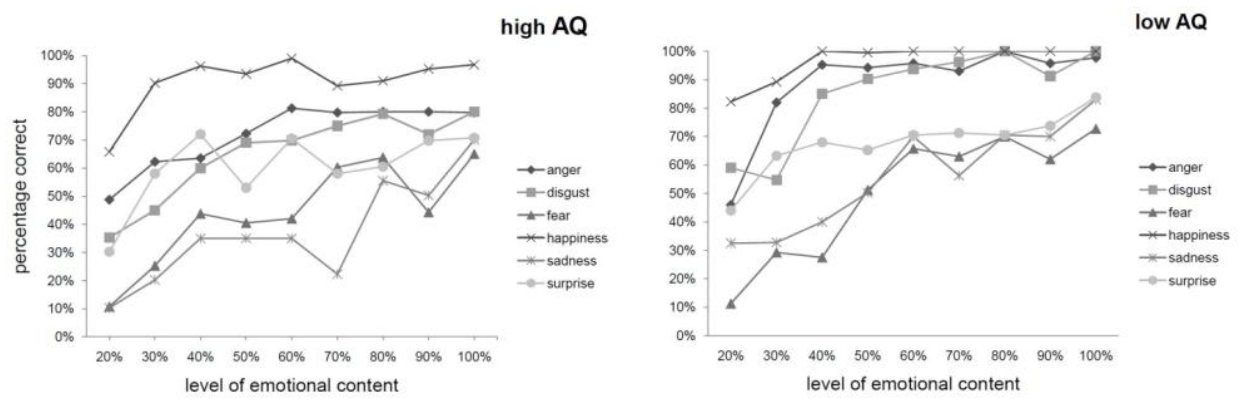

Figure 2. The accuracy of recognition plotted for all levels of emotional intensity (xaxis, levels 1-9 represent 20-100\% emotional content in the face). A general trend for all emotions is an increase in the recognition rate associated with an increase of the emotional content. In the high AQ group, emotions of anger, disgust, happiness and sadness have a lower recognition rate compared to the low AQ group.
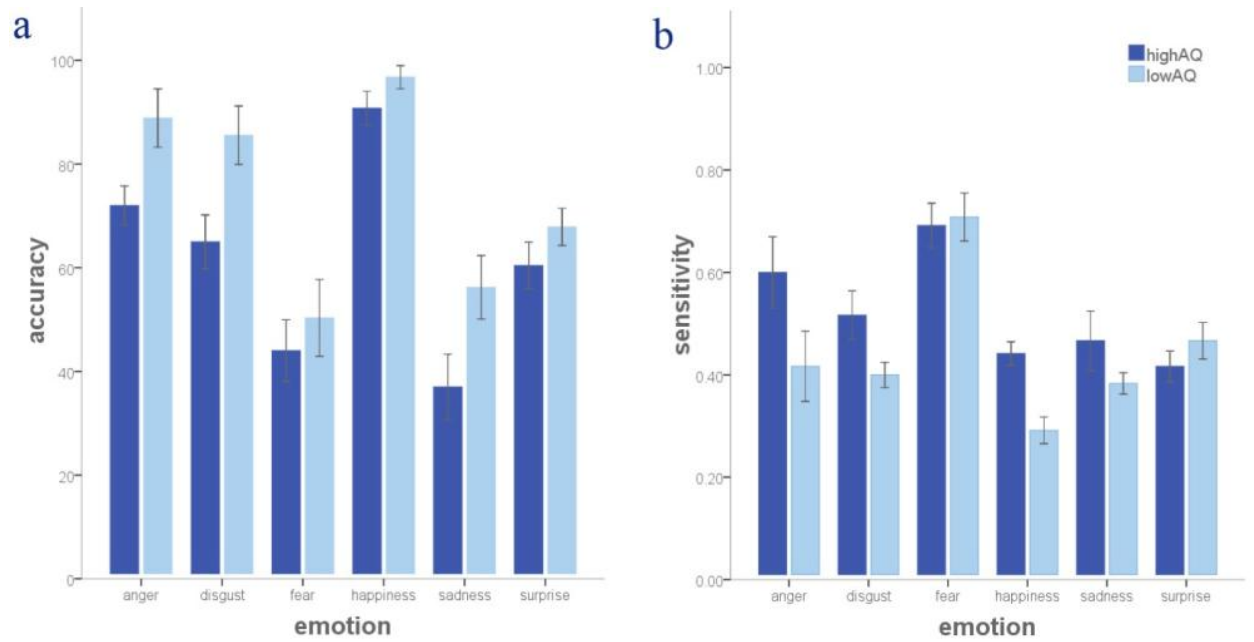

Figure 3. The accuracy of responses for the two groups (a) and the level of emotional content necessary to correctly identify each emotion (b). The pattern resembles the findings in previous studies, the emotion of happiness requiring lowest amount of emotional content to recognize it correctly while the emotion of fear is most difficult to identify. There are significant differences between the two groups for the emotions of anger, disgust, happiness and sadness. Error bars represent 1 SE. 


\section{Discussion}

The current study investigated the recognition of facial emotional expressions in people with high Autism-spectrum Quotient. The findings showed a decreased sensitivity for the emotions of anger, disgust, happiness, and sadness. The high AQ group needed more intense emotional expressions compared to the group that had low AQ-scores to correctly identify the facial emotions. In addition, a reduced accuracy was also observed in the high-AQ group for the same emotions, anger, disgust, happiness, and sadness.

The findings of the present study are in accordance with previous studies that showed impaired recognition of fear, disgust and sadness in other patient populations with disorders in the area of affective functioning (Montagne et al., 2005, 2006a, 2006b; Poljac, Montagne \& de Haan, 2010), as well as with the findings of Law Smith, Montagne, Perret, Gill, and Gallagher (2010) showing different recognition of disgust, anger and surprise. Impaired processing of the emotion of disgust has been previously reported in adults with Autism spectrum disorders (Ashwin, Chapman, Colle, \& Baron-Cohen, 2006; Ellis \& Leafhead, 1996). Beside the shared characteristics of the high AQ score group and autistic individuals, a number of possible alternative explanations of the findings must be discussed.

First, deficits in basic face processing can certainly influence the perception of emotions in faces. The neural systems that mediate face processing develop very early in life. Babies already respond differently to familiar faces. Responses (ERP's) to familiar and unfamiliar faces of children with autism do not differ, which indicates an impaired ability to process faces (Dawson, Webb, Carver, Panagiotides, \& McPartland, 2004). However, the observed compromised performance in the high AQ group in this study is most likely not related to basic deficits in the perception of faces, as both groups had normative scores and did not differ on the Benton Facial Recognition Test.

Another possible cause of dissimilar processing of emotional expressions might be sought in the level of general cognitive functioning. The ability to recognize emotions usually correlates with general cognition (Mathersul et al., 2009). Montagne et al. (2005) observed a 
relationship between cognitive performance and the performance on The Emotion Recognition Task in post-stroke depression (PSD) patients. A number of studies have linked autism with impairments in cognitive functioning, in particular with impairments in the area of social cognition. For instance, in their recent study Coutureet al. (2010) show that individuals with high-functioning autism were impaired on a variety of social cognitive tasks relative to the non-clinical controls.

Deficits in social cognition are frequently related to a deficient theory of mind (ToM), but also to problems detecting emotions in social stimuli, which in turn has been identified as likely contributor to social dysfunction (e.g. Muris et al. 1999; Couture et al. 2006; Crespi \& Badcock, 2008). ToM theory predicts that individuals with ASD have difficulties processing emotional expressions that require the ability to represent the mental states of others (Baron-Cohen et al., 1993). BaronCohen, Spitz, and Cross (1993) used this theory is to explain difficulties amongst children with ASD in processing the expression of surprise. Impaired perception of basic emotion perception in particular correlates to higher-order ToM tasks (Baron-Cohen, O'Riordan, Stone, Jones, \& Plaisted, 1999; Kaland et al. 2002; Brune, 2005).

Although cognitive functioning was not assessed in the present study, this is an unlikely explanation for the observed dissimilarities, because the two groups did not differ in their abilities to detect facial identity in the Benton task (a cognitive task) and it is difficult to see why cognitive decline would result in a selective problem for anger, disgust, happiness and sadness. On the other hand, perception of facial expressions is believed to occur independently from other aspects of face processing (De Haan, 2002; Hargrave, Maddock, \& Stone, 2002). Patients with prosopagnosia have difficulties in identifying faces, but their recognition of emotions may remain intact (Stephan, Breen, \& Caine, 2006). The selective disruption of the ability to identify certain emotions has been described before (McClure et al., 2003; Venn et al., 2006). For instance, patients with damage to the amygdale may be impaired in the perception of anger and fear, while insular lesions appear to selectively affect the recognition of disgust (Adolphs et al., 1994). There is now converging evidence from neuroimaging studies suggesting 
separate neural substrates underlying the recognition of different facial (emotional) expressions (Phan, Wager, Taylor, \& Liberzon, 2002). Selective deficits have also been observed in psychiatric populations, such as the social anxiety disorder, which is characterized by an attentional avoidance of angry facial expressions (McClure et al., 2003). Anatomical differences (and their functional consequences) represent another possible cause of the different performance of the present study found for the specific emotions. As there are no anatomical data for the participants, it is speculative, yet not unreasonable to suggest a role for the amygdala. Impaired processing of emotions is commonly attributed to limbic system, in particular the basal ganglia, amygdale, the insula, the cingulate, and the medial frontal lobe (Frewen, Dozois, Neufeld \& Lanius, 2008; Dolan, 2009). Altered amygdala activity has also been observed in other types of disorders, such as psychopathic individuals. Amygdala damage has been associated with a selective impairment of fear recognition (Rauch et al., 2006), and Venn et al. (2006) refer to a number of studies in support of a possible link between the amygdala and sadness perception.

Even though participant's emotional state can influence the perception and interpretation of facial emotional expressions, it is unlikely that the found differences are related to differences in emotional state in this study. Montagne et al. (2005) showed decreased overall accuracy in the recognition of facial expressions in people with depressive symptoms after a stroke. However, the BDI scores for the two groups were similar, and in addition, the pattern of performance with a selective deficit for certain emotions and not for others has not been related to emotional state (depression for instance, Montagne et al. 2005).

The present findings may possibly have significant clinical implications. The assessment of emotion perception could become a clinically useful additional tool to neurocognitive assessment of autistic patients. Impairments in affective processing in Huntington disease (HD), such as deficit in the recognition of disgust, have been observed even in the pre-symptomatic stage when motor deficits are not yet present (Speedie, Brake, Folstein, Bowers, \& Heilman, 1990). A similar suggestion for the measurement of facial expression perception as a 
useful tool for assessing the efficacy of antidepressant treatments was made by Venn et al. (2006). The high AQ individuals show a very specific emotion recognition deficiency that might be characteristic pattern for autism related disorders. If this is true, then the emotion recognition task may be a useful tool for assessing the possible existence of autism, when other symptoms or instruments cannot provide clear answers. Moreover, the observed differences were identified at lower levels of emotional intensity, but when the emotion was fully displayed in the face, these differences were not present anymore. This means that ERT is a very sensitive for detecting subtle differences at lower intensity levels. In addition, this tool could be used to improve prognostic models for individual differences in treatment outcome of patients with autism.

In sum, the current study demonstrated that a decreased sensitivity of individuals with a high AQ score for the emotions of anger, disgust, happiness and sadness, as well as lower accuracy for these emotions, as perceived from facial expressions. This selective impairment might be helpful in trying to understand the psychopathology of autism spectrum disorders. In addition, the Emotion Recognition Task might be used in the clinical practice for assessing autism and as well as the development of more detailed prognostic models. 


\section{References}

Adolphs R, Tranel D, Damasio H, and Damasio AR. (1994) Impaired recognition of emotion in facial expressions following bilateral damage to the human amygdala. Nature, 372: 669-672.

American Psychiatric Association (2000) Diagnostic and statistical manual of mental disorders. DSM-IV-TR (text revision), 4th edn. American Psychiatric Press, Washington, DC.

Ashwin C, Chapman E, Colle L, and Baron-Cohen S. (2006). Impaired recognition of negative basic emotions in autism: A test of the amygdala theory. Social

Neuroscience, 1: 349-363.

Baron-Cohen, S. (1993). From attention-goal psychology to belief-desire psychology: the development of a theory of mind, and its dysfunction. In S. Baron Cohen, H. Tager-Flusberg \& D. Cohen (Eds.), Understanding other minds: Perspectives from autism (pp. 59-82). Oxford: Oxford University Press.

Baron-Cohen S, Hoekstra RA, Knickmeyer R, and Wheelwright S. (2006) The Autism Spectrum Quotient (AQ)-Adolescent version. Journal of Autism and Developmental Disorders, 36(3): 343-350

Baron-Cohen S, Jolliffe T, Martimore C, and Robertson M. (1997). Another advanced test of theory of mind: Evidence from very high functioning adults with autism or Asperger syndrome. Journal of Child Psychology and Psychiatry, 38: 813-822.

Baron-Cohen S, O'Riordan M, Stone V, Jones R, Plaisted K (1999). Recognition of faux pas by normally developing children with Asperger syndrome or high functioning autism. Journal of Autism and Developmental Disorders 29: 407-418.

Baron-Cohen S, Ring H, Wheelwright S, Bullmore E, Brammer, M, Simmons A, \& Williams S. (1999). Social intelligence in the normal and autistic brain: an fMRI study. European Journal of Neuroscience, 11: 1891-1898.

Baron-Cohen S, Spitz A, and Cross P. (1993). Can children with autism recognize surprise? Cognition and Emotion, 7: 507-516. 
Baron-Cohen, S., Tager-Flusberg, H., \& Cohen, D. (Eds.). (1993). Understanding other minds: Perspectives from autism. Oxford, UK: Oxford University Press.

Beck AT, Ward CH, Mendelson M, Mock J, and Erbaugh J. (1961) An inventory for measuring depression. Archives of General Psychiatry, 4: 561 - 71

Benton AL, Sivan AB, deS Hamsher K, Varney NR, and Spreen, O. (1994). Contributions to neuropsychological assessment. A clinical manual (2nd ed.). New York: Oxford University Press.

Bolte S, and Poustka F. (2003). The recognition of facial affect in autistic and schizophrenic subjects and their first degree relatives. Psychological Medicine,

33: 907-915.

Brune M (2005). 'Theory of mind' in schizophrenia: A review of the literature. Schizophrenia Bulletin, 31: 21-42.

Celani G, Battacchi MW, and Arcidiacono L. (1999). The understanding of the emotional meaning of facial expressions in people with autism. Journal of Autism and Developmental Disorders, 29: 5766.

Couture SM, Penn DL, Losh M, Adolphs R, Hurley R, and Piven J. (2010) Comparison of social cognitive functioning in schizophrenia and high functioning autism: more convergence than divergence. Psychological Medicine, 40(4): 569-579.

Crespi B and Badcock C (2008). Psychosis and autism as diametrical disorders of the social brain. Behavioral and Brain Sciences 31: 241-320.

Davies S, Bishop D, Manstead ASR, and Tantam D. (1994). Face perception in children with autism and Asperger's syndrome. Journal of Child Psychology and Psychiatry, 35: 1033-1057.

Dawson G. (1996). Neuropsychology of autism: a report on the state of the science. Journal of Autism and Developmental Disorders, 26, 179-184.

Dawson G, Meltzoff AN, Osterling J, Rinaldi J, \& Brown E. (1998). Children with autism fail to orient to naturally occurring social 
stimuli. Journal of Autism and Developmental Disorders, 28, 479485.

Dawson G, Webb S, Carver L, Panagiotides H, and McPartland J. (2004).

Young children with autism show atypical brain responses to fearful versus neutral facial expressions. Developmental Science, 7: 340-359.

De Haan EHF. (2002) Prosopagnosia. In Ramachandran (Ed.). Encyclopedia of the Human Brain. New York: Elseviers Science. p. 67-74.

Dolan, R. J. (2009). The anatomy of human emotion. In Gelder, M. G., Andreasen, N. C., Lopez-ibor, J. J., Geddes, J. R. (Eds.). New Oxford Textbook of Psychiatry (2nd ed. pp.257-262). Oxford: Oxford University Press.

Frewen PA, Dozois DJA, Neufeld RWJ and Lanius RA. (2008) Metaanalysis of alexithymia in posttraumatic stress disorder. Journal of Traumatic Stress, 21: 243-246.

Hargrave R, Maddock RJ and Stone V. (2002) Impaired recognition of facial expressions of emotion in Alzheimer's disease. The Journal of Neuropsychiatry and Clinical Neurosciences, 14: 64-71.

Ellis HD and Leafhead KM. (1996). Raymond: A study on an adult with Asperger syndrome. In PW Halligan \& JC Marshall (Eds.), Method in madness: Case

studies in cognitive neuropsychiatry. Hove: Psychology Press.

Ekman P. Basic Emotions. In Dalgleish T and Power M (Eds.). (1999) Handbook of cognition and emotions. Sussex, UK: Wiley \& Sons.

Frith, U. (1989). Autism: Explaining the enigma. Oxford: Blackwell. Gauthier, I., Tarr, M.J., Anderson, A.W., Skudlarski, P., \& Gore, J.C. (1999). Activation of the middle fusiform 'face area' increases with expertise in recognizing novel objects. Nature Neuroscience, 2, 568-573.

Green RE, Turner GR, and Thompson WF. (2004) Deficits in facial emotion perception in adults with recent traumatic brain injury. Neuropsychologia, 42: 133-41.

Grossman JB, Klein A, Carter AS, and Volkmar FR. (2000). Verbal bias in recognition of facial emotions in children with Asperger 
syndrome. Journal of Child Psychology and Psychiatry, 41: 213225.

Kaland N, Moller-Neilsen A, Callesen K, Mortensen EL, Gottlieb D, AND Smith L (2002). A new 'advanced' test of theory of mind: evidence from children and adolescents with Asperger syndrome. Journal of Child Psychology and Psychiatry, 43: 517-528.

Kleinhans NM, Johnson LC, Richards T, Mahurin R, Greenson J, Dawson G, Aylward E. (1999) Reduced neural habituation in the amygdala and social impairments in autism spectrum disorders. American Journal of Psychiatry, 166: 467-475.

Law Smith MJ, Montagne B, Perret DI, Gill M, and Gallagher L. (2010) Detecting subtle facial emotion recognition deficits in highfunctioning Autism using dynamic stimuli of varying intensities. Neuropsychologia, 48: 2777-2781.

Lembke A and Ketter TA. (2002) Impaired recognition of facial emotion in mania. American Journal of Psychiatry, 159: 302-304.

Mathersul D, Palmer DM, Gur RC, Gur RE, Cooper N, Gordon E, and Williams LM. (2009) Explicit identification and implicit recognition of facial emotions: II. Core domains and relationships with general cognition Journal of Clinical and Experimental Neuropsychology, 31: 278-291.

McClure EB, Pope K, Hoberman AJ, Pine DS, and Leibenluft E. (2003) Facial expression recognition in adolescents with mood and anxiety disorders. American Journal of Psychiatry, 160: 1172-4.

Montagne B, Kessels RP, De Haan EH, and Perrett DI. (2007) The Emotion Recognition Task: A paradigm to measure the perception of facial emotional expressions at different intensities. Perceptual and Motor Skills, 104: 589-598.

Montagne B, Kessels RPC, Frigerio E, De Haan EHF and Perret DI. (2005) Sex differences in the perception of affective facial expressions: Do men really lack emotional sensitivity? Cognitive Processing, 6: 136-141.

Montagne B, Kessels RPC Wester AJ and de Haan EHF. (2006a) Processing of emotional facial expressions in Korsakoff's syndrome. Cortex, 42: 705-710. 
Montagne B, Schutters S, Westenberg HG, Kessels RP, and de Haan EH. (2006b) Reduced sensitivity in the recognition of anger and disgust in social anxiety disorder. Cognitive Neuropsychiatry, 11: 389-401.

Muris P, Steerneman P, Meesters C, Merckelbach H, Horselenberg R, van den Hogen T, van Dongen L (1999). The TOM test : a new instrument for assessing theory of mind in normal children and children with pervasive developmental disorders. Journal of Autism and Developmental Disorders 29: 67-80.

Ogai M, Matsumoto H, Suzuki K, Ozawa F, Fukuda R, Uchiyama I, et al. (2003). fMRI study of recognition of facial expressions in highfunctioning autistic

patients. Neuroreport, 14: 559-563.

Phan KL, Wager T, Taylor SF, and Liberzon I. (2002) Functional Neuroanatomy of Emotion: A Meta-Analysis of Emotion Activation Studies in PET and fMRI, NeuroImage, 16: 331-348.

Rauch SL, Shin LM, and Phelps EA. (2006) Neurocircuitry models of posttraumatic stress disorder and extinction: human neuroimaging research-past, present and future. Biological Psychiatry, 60: 376382.

Rosset DB, Rondan C, Da Fonseca D, Andreia Santos, Assouline B, and Deruelle C. (2008) Typical emotion processing for cartoon but not for real faces in children with Autistic Spectrum Disorders. Journal of Autism and Developmental Disorders, 38: 919-925.

Speedie LJ, Brake N, Folstein SE, Bowers D, and Heilman KM. (1990) Comprehension of prosody in Huntington's disease. Journal of Neurology, Neurosurgery \& Psychiatry, 53: 607-610.

Stephan BC, Breen N and Caine D. (2006) The recognition of emotional expression in prosopagnosia: Decoding whole and part faces. Journal of the International Neuropsychological Society, 12: 884895, 2006

Venn HR, Gray JM, Montagne B, Murray LK, Michael Burt D, Frigerio E, Perrett DI, and Young AH. (2004) Perception of facial expressions of emotion in bipolar disorder. Bipolar Disorders, 6: 286-93. 